\title{
ORIENTATION DE LA FILAMENTISATION DES CHAMPIGNONS LEVURIFORMES CULTIVÉS SUR LAMES GÉLOSÉES
}

\author{
Par Maurice LANGERON et Paul GUERRA
}

Lorsqu'on ensemence en trois stries parallèles, sur une lame gélosée, un champignon levuriforme (Candida) à filamentisation rapide et facile, tel par exemple que Candida tropicalis, on observe une très curieuse inégalité de développement (fig. 2 et 30 ) :

$1^{\circ}$. - La strie médiane filamente peu ou même pas du tout.

$2^{\circ}$. - Les stries latérales développent des filaments sur leur côté externe et peu ou pas du côté interne.

Nous employons toujours cette méthode des trois stries parallèles pour l'étude de la morphologie des Candida qui donnent une filamentisation trop abondante et touffue. Nous obtenons ainsi, sur la strie médiane et sur le côté interne des stries latérales, des filaments bien dégagés, par conséquent plus faciles à étudier et à photographier. Pour C. tropicalis c'est ainsi que l'on voit apparaître la morphologie caractéristique avec chaînettes de pseudo-conidies (cf. Langeron et Guerra, 1938 , p. 460 , pl. XVIII, fig. 3 et 4 , et pl. XIX, fig. 1-3).

Nous avons poursuivi pendant assez Iongtemps une série d'expériences, dans le but d'élucider la nature et le déterminisme de ce phénomène.

I. Zone d'extension maxima de la filamentisation. - Une première constatation est que l'extension maxima de la filamentisation se trouve toujours du côté où il y a la plus grande surface libre de milieu (fig. 1).

II. Influence réciproque des stries d'ensemencement. - Un autre fait est que les stries exercent une influence très nette les unes sur les autres, quand elles sont suffisamment rapprochées. Cette influence arrête la croissance du champignon en général et se manifeste particulièrement sur la filamentisation. En effet, du côté influencé, les filaments peuvent ne pas apparaitre ou bien, s'ils se développent, ils sont plus espacés et plus courts. Ils peuvent même

Annales de Parasitologie, T. XVII, No $6 .-1939-1940$, p. 580-589. 
présenter une tendance à se réfléchir sur la strie qui leur a donné naissance, tendance manifestée par le renversement des verticilles.

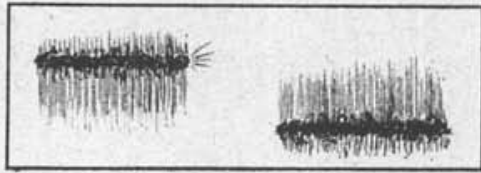

Fig. 1: - Orientation générale de la filamentisation

7 vers le maximum d'espace libre

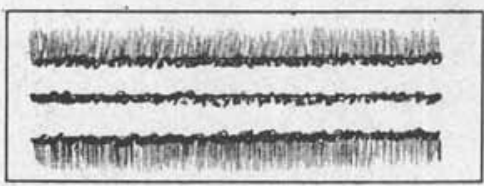

Fıg. 2. - Influence réeiproquue des stries d'ensemencement parallèles et rapprochées. La strie médiane ne produit aucun filament : il en est de mćme pcur les deux stries latérales, du côté quii regarde la strie médiane.

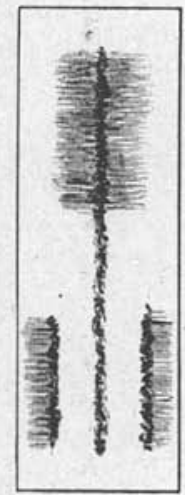

3

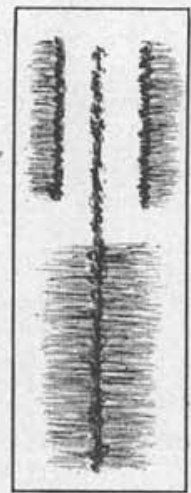

4

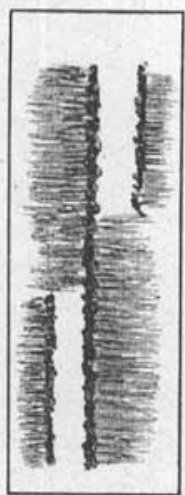

5

F1G. 3, 4, 5. - Raccourcissement et variations de position des stries latérales pour démontrer la réalité de l'influence réciproque des stries d'ensemencement

La fig. 2 montre l'influence réciproque de trois stries d'ensemencement tracées parallèlement sur une lame gélosée. La strie médiane ne filamente pas et elle empêche les deux stries latérales de filamenter sur leur côté interne. 
Une autre disposition, destinée à confirmer ce phénomène d'influence réciproque, est représentée par les fig. $3,4,5$, où les stries latérales sont raccourcies de plus de moitié et disposées de trois manières différentes par rapport à la strie médiane; celle-ci ne peut donner de filaments qu'en face des espaces libres et couverts de gélose.

La fig. 6 montre l'influence réciproque de stries non parallèles : plus les stries se trouvent rapprochées, plus l'influence empêchante

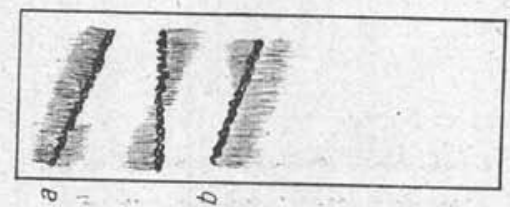

Fig. 6. - Inclinaison des stries d'ensemencement: l'influence est inversement proportionnelle à la distance qui les sépare. Phénomène de barrage preduit par la strie : sur le côté externe des stries $a$ et $b$, la filamentisation est uniformément développée.

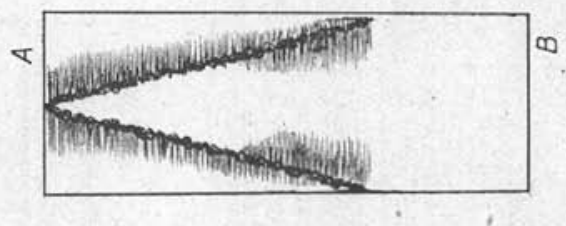

Fig. 7. - Disposition angulaire de deux stries d'ensemencement: l'influence est inversement proportionnelle à l'ouverture de l'angle, c'est-à-dire à la distance des stries.

est nette. Elle révèle en outre ce fait très curieux que la strie d'ensemencement se comporte comme une barrière et arrête toute influence : en effet, les filaments externes des stries $a$ et $b$ sont également développés sur touie la longueur de ces stries.

La fig. 7 montre, encore mieux, que l'influence réciproque est inversement proportionnelle à la distance qui sépare les stries ; dans la région du sommet de l'angle les stries s'empêchent réciproquement de filamenter ; à mesure qu'elles s'écartent, la filamentisation apparait.

$\mathrm{Si}$, avec cette même orientation des stries à angle aigu, on prépare plusieurs lames portant des couches de gélose d'épaisseur différente, on remarque que plus la couche est épaisse, moins l'influence empê- 
chante se manifeste et plus la filamentisation se rapproche du sommet A de l'angle. La concentration du milieu en matériaux nutritifs agit dans le même sens que l'épaisseur ; donc, plus le milieu est riche (cians certaines limites), plus la filamentisation se rapprochera du sommet A.

III. Conditions du phénomène d'influence. - Le phénomène d'influence réciproque est indépendant de la position verticale ou horizontale des lames, ainsi que de la lumière. L'humidité et la séche-

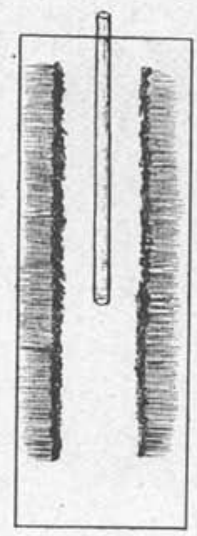

8

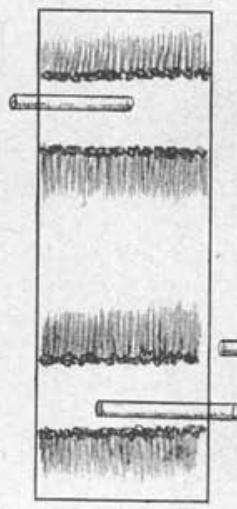

9

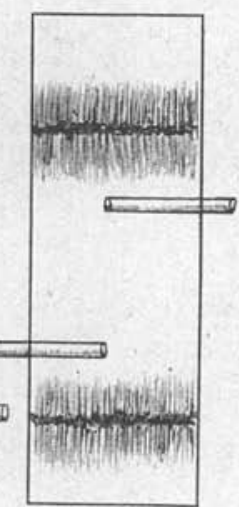

10

Fig. 8-10, - Influence négative d'une tige de verre pleine ou creuse : en 8 et 9 , l'action empêchante réciproque des stries se produit comme si aucun objet n'était interposé ; en 10 (témoin), les stries étant très écartées, la filamentisation bilatérale est normale.

resse, dans la mesure où elles ne sont pas nuisibles à la filamentisation, n'ont aucune influence sur le phénomène. Il en est de même de l'épaisseur de la couche de gélose qui peut affaiblir le phénomène, mais ne l'inhibe pas.

Il est nécessaire que la strie soit formée de cellules vivantes : le dépôt de masses, même considérables, de blastospores tuées n'exerce aucune influence sur la filamentisation.

L’influence réciproque empêchante est nettement indépendante de la nature du champignon. Ainsi, les stries des figures 1-7 ont été faites avec Candida tropicalis (souche $255 \mathrm{~A}$, Mycothèque de l'Institut Oswaldo Cruz à Rio de Janeiro). Nous avons répété les mêmes expériences en combinant sur la même lame :

C. tropicalis $(255 \mathrm{~A})$ et C. parakrusei. 
C. tropicalis (255 A) et C. albicans (souche $403 \mathrm{~A}$ à colonies membraneuses et à filamentisation très facile).

C. tropicalis (255 A) et Rhodotorula mucilaginosa.

C. tropicalis (255 A) et un Hormodendron.

C. tropicalis (255 A) et un Trichosporum (souche $398 \mathrm{~A}$, piedra du Japon).

Toutes ces expériences ont donné les mêmes résultats, mais à des degrés différents : plus la strie est épaisse, c'est-à-dire plus elle renferme de cellules, plus l'influence empêchante est puissante. . -

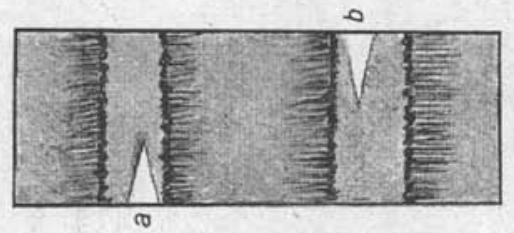

Frg. 11. - Le grattage d'un petit triangle de gélese n'a pas arrêté l'action empêchante

IV. Action d'une tige de verre pleine ou creuse. - L'influence réciproque empêchante n'est ni arrêtée ni diminuée par l'interposi-

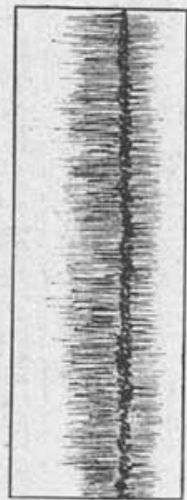

a

12

$b$

F16. 12. - Entre deux́ lames juxtaposées, l'action empéchante ne s'exerce pas. En $a$, la filamentisation est normale, son orientation est celle de la fig. 1 ; en $b$, la petite strie inductrice, même ensemencée au bord de la lame, n'exerce aucune action sur $a$. tion d'une tige de verre pleine ou creuse, telle qu'une baguette de verre ou une effilure de pipette, comme le montrent les figures 8 et 9 . La lame $\mathrm{n}^{\circ} 10$ est un témoin destiné à montrer que ces tiges de verre sont sans influence sur la filamentisation.

V. Action d'une solution de continuité dans le milieu de culture. Une solution de continuité dans le milieu de culture arrête en général l'influence empêchante. Toutefois, cette condition est assez difficile à contrôler car la limite du milieu agit un peu comme une strie empêchante.

A. Dans la lame représentée par la figure 11, nous avons enlevé avec un scalpel deux petits triangles de gélose $a$ et $b$. On voit qu'aucun changement ne s'est produit dans la filamentisation en face de ces triangles vides. 
B. Au contraire, lorsque deux lames sont juxtaposées et séparées par une très faible distance, l'influence empêchante est arrêtée. Dans la fig. 12, la lame $a$ est ensemencée d'une grande strie, tandis que la lame $b$ ne porte qu'une petite strie inductrice dans sa partie médiane : aucune influence ne s'est manifestée sur la strie de la lame $a$.

VI. Voies de conduction de l'influence empêchante. - Des expériences représentées par les fig. 8-12, nous pouvons conclure :

$1^{\circ}$ que l'influence empêchante ne se transmet pas par l'air ;

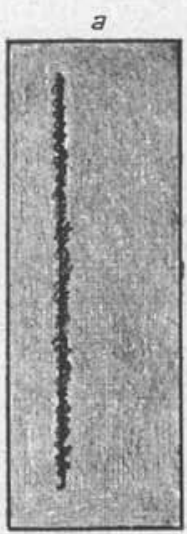

13

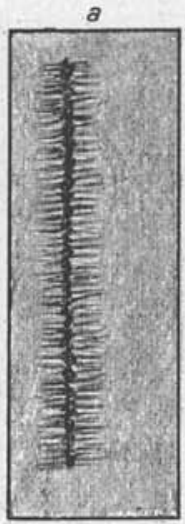

14

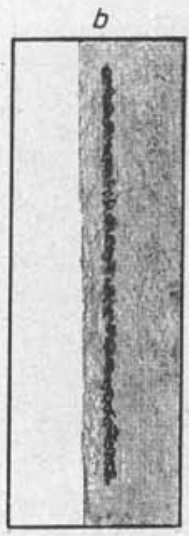

15

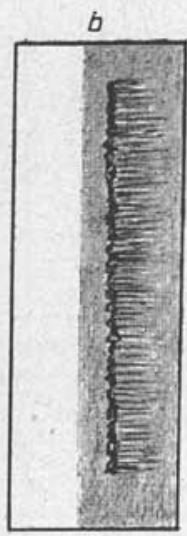

16

F1G. 13-16. - Stries parallèles. En 13, ensemencement de la strie a du côté gauche de la lame; cette strie s'est développée en 14. En 15, on a enlevé autant que possible toute la strie $a$, ainsi que la gélose, puis à droite on ensemence une nouvelie strie $b$. En 16 , la strie $b$ ne filamente pas cn face de la zone où s'était développée la-strie $a$.

$2^{\circ}$ qu'elle se transmet à travers le milieu de culture.

Il ne s'agit done pas d'une radiation (mitogénétique ou autre) comme on aurait pu ètre tenté de le penser.

VII. Nature de l'influence empêchante. - Aux deux conclusions précédentes nous sommes amenés à en ajouter une troisième : c'est que l'influence empêchante est due à une modification subie par le milieu de culture au cours du développement de la strie d'ensemencement et de la pullulation de blastospores qui précède la filamentisation. C'est cette modification du milieu et non la présence du champignon vivant qui produit cette influence sur l'orientation de la filamentisation. C'est ce que les expériences suivantes vont prouver : 
a. Stries parallèles, - Lès fig. 13 à 16 représentent une série de 4 lames. Sur la lame 13 on ensemence une strie (a) qu'on laisse se développer jusqu'à filamentisation (fig. 14). On enlève alors avec un scalpel la strie $a$ (fig. 15) et on ensemence une nouvelle strie $b$ qui ne filamente jamais du côté où la strie $a$ a été ensemencée (fig. 16).

b. Stries perpendiculaires, - Sur une lame (fig. 17) on ensemence une strie transversale $a$ qu'on laisse se développer jusqu'à filamentisation (fig. 18). On l'enlève au scalpel (fig. 19) et on trace une

a

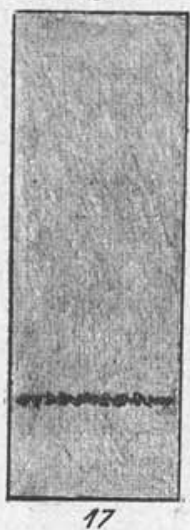

$a$

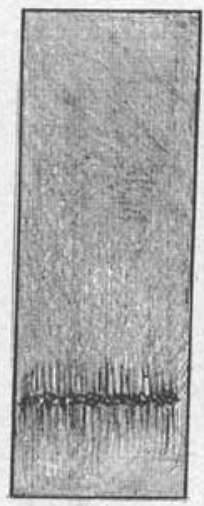

18

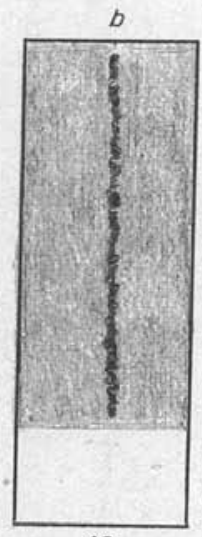

19

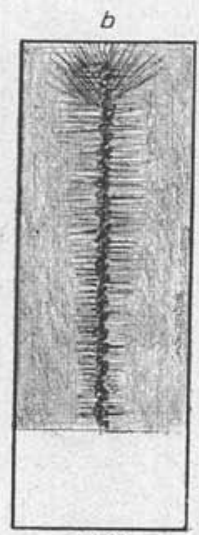

20

Fig. 17-20. - Stries perpendiculaires. Même expérience que pour 13-16, mais la strie induite $b$ est perpendiculaire à la strie inductrice $a$. Fn 20, la strie $b$ filamente d'autant mieux qu'elle s'éloigne plus de la zone où s'était développée $a$.

strie $b$ (fig. 19) perpendiculaire à la direction de l'ancienne strie $a$. $\mathrm{Au}$ bout de quelques jours, la strie $b$ développe sa filamentisation, mais celle-ci s'atténue en pointe à l'extrémité inférieure tandis qu'elle s'épanouit en éventail à l'extrémité supérieure (fig. 20).

c. Stries verticales multiples parallèles. - Sur une lame (fig. 21) on trace deux stries $a$ qui s'arrêtent à la moitié de la hauteur de la lame. Après filamentisation (fig. 22) on les enlève au scalpel (fig. 23) et on trace dans l'espace intermédiaire une nouvelle strie $b$ (fig. 23) qui filamente dans sa moitié supérieure, mais non dans sa moitié inférieure (fig. 24).

d. Stries transversales interrompues. - Sur une lame gélosée sur sa partie médiane (fig. 25) on trace deux stries transversales parallèles $a$ et $b$, qu'on laisse filamenter (fig. 26). On enlève alors au 
scalpel une partie de la strie $b$ (fig. 27) : aucun changement ne se produit dans la strie $a$ (fig. 28). Mais, si on dépose en $c$ une trace de

a

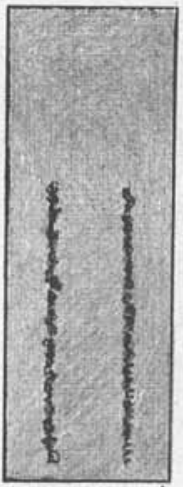

21

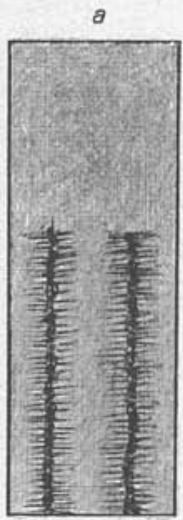

22

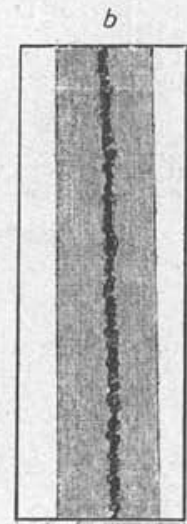

23 $b$

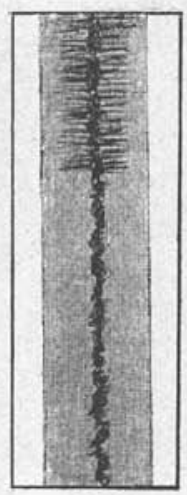

24

Fig. 21-24. - Stries parallèles multiples. Mème résultat que pour 13-16. Mème après enlèvement des stries $a$, la strie $b$ ne filamente pas en face des zones le long desquelles les stries, $a$ s'étaient développées.

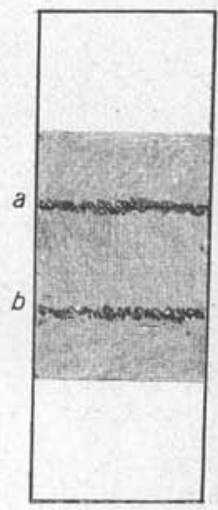

25

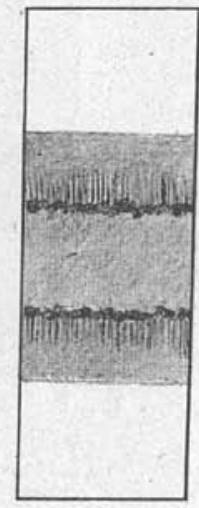

26

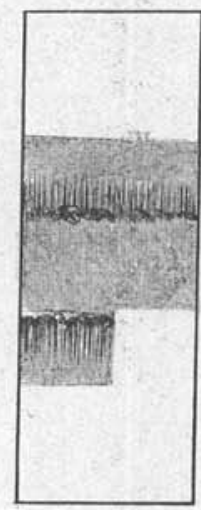

27

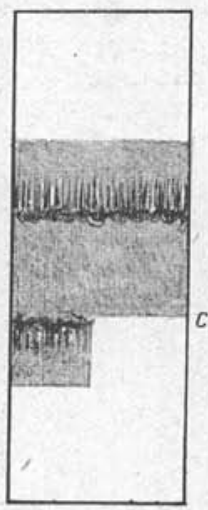

28

Fig. 25-28. - Stries transversales interrompues. - Les lames ne sont gélosées qu'au milieu. Après développement de la filamentisation, l'ablation d'une partie de la strie $b$ n'amène pas la filamentisation de Ia partie correspondante de $a$; toutefois, si on dépozc en $c$ vne trace de glycose, on peut provoquer l'apparition de quelques filements.

glycose, on peut voir apparaître une très légère filamentisation du côté interne de la strie $a$.

La modification subie par le milieu de culture et révélée par ces 
expériences peut provenir soit d'une soustraction d'aliments essentiels (comme le montre l'action d'une trace de glycose), soit de la diffusion de substances inhibitrices produites au niveau des stries empêchantes, soit enfin de ces deux actions combinées.

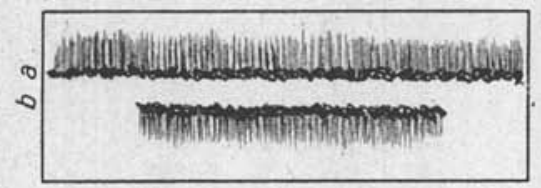

Fig. 29. - Exemple du barrage produit par les stries : les stries $a$ et $b$ s'influencent réciproquement et ne filamentent pas en dedans, mais les filamentisations externes sont normales, même dans la partie où les deux stries sont en face l'une de l'autre.

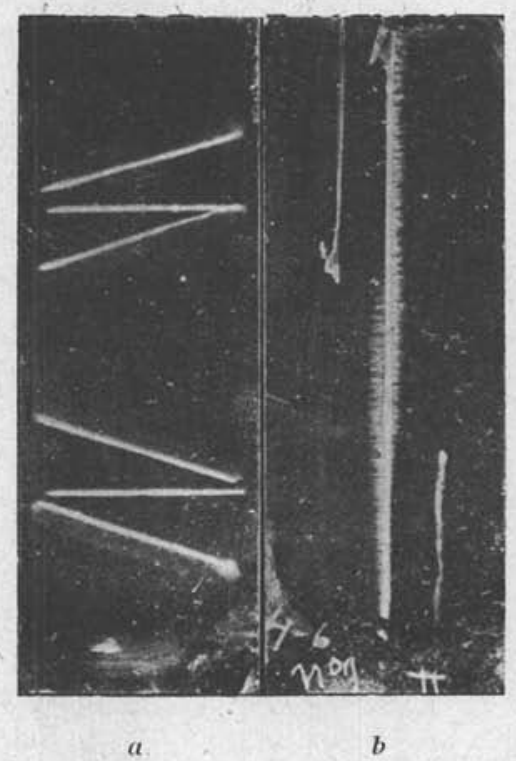

F1G. 30. - Aspect réel des expériences d'orientation avec Candida tropicalis : . $a$ correspond à la fig. 6 et $b$ à la fig. 5 .

La diffusion de substances solubles dans la gélose, surtout la gélose glycosée comme l'a montré Boysen Jensen (1933) (1), est un

(1) Ueber den Nachweis von Wuchsstoff in Wurzeln. Planta, XIX, 1933, p. $345-350$, 
phénomène bien connu et largement utilisé pour l'étude des facteurs de croissance.

Dans nos expériences les phénomènes de diffusion doivent être très accentués puisque, même après ablation de la strie et de la gélose qui la supporte (fig. 13-24), il reste encore assez de substance diffusible pour inhiber la filamentisation.

Mais la diffusion est arrêtée net par le barrage de la strie (fig. 6 et 29), même avec des stries de longueur inégale (fig. 29) : du côté externe, la filamentisation est toujours normale. Il est possible qu'il s'agisse d'un phénomène d'électrophorèse résultant d'une différence de potentiel suffisante pour arrêter la diffusion au niveau de la strie formée d'une accumulation de cellules vivantes.

\section{RÉSUMÉ}

Dans les cultures de champignons levuriformes en stries multiples sur lames gélosées, nous avons observé que les stries, lorsqu'elles sont insuffisamment éloignées, exercent les unes sur les autres deux actions qui règlent l'orientation de la filamentisation :

$1^{\circ}$ une action empêchante qui diminue ou même inhibe complètement la filamentisation ;

$2^{\circ}$ un effet inverse de barrage, qui arrête totalement l'influence empêchante, celle-ci ne pouvant traverser les stries et s'exercer de l'autre côté, sur lequel la filamentisation est normale.

Une série de figures schématiques représente les modalités de nos expériences.

La conclusion est que l'action empêchante est due exclusivement à l'activité de cellules vivantes, mais est indépendante de la nature du champignon. Elle résulte de modifications du milieu gélosé produites : soit par diffusion de substances inhibitrices naissant au niveau de la strie, soit par soustraction d'aliments nécessaires par suite du développement du champignon, soit enfin par ces deux causes réunies. Il en résulte une sorte de vaccination du milieu qui arrête ou diminue considérablement la filamentisation dans la zone qui sépare deux stries.

Institut de parasitologie de la Faculté de médecine de Paris (Directeur : Prof. E. Brumpt), Section de mycologie médicale (Chef de service: $D^{r}$ Maurice Langeron). 